\title{
DEPOSIÇÃO DE SERAPILHEIRA E NUTRIENTES EM ÁREAS DE MINERAÇÃO SUBMETIDAS A MÉTODOS DE RESTAURAÇÃO FLORESTAL EM PARAGOMINAS, PARÁ
}

\author{
Walmer Bruno Rocha Martins ${ }^{1 *}$, Gracialda Costa Ferreira ${ }^{1}$, Fernanda Pantoja Souza ${ }^{1}$, Luiz Fernandes Silva \\ Dionísio $^{1}$, Francisco de Assis Oliveira ${ }^{1}$ \\ ${ }^{1}$ Universidade Federal Rural da Amazônia, Instituto de Ciências Agrárias, Belém, Pará, Brasil - walmerbruno@gmail.com.br*; \\ gracialda.ferreira@ufra.edu.br; fernandap_souza@yahoo.com.br; fernandesluiz03@gmail.com; francisco.oliveira@ufra.edu.br
}

Recebido para publicação: 17/11/2016 - Aceito para publicação: 23/01/2018

\begin{abstract}
Resumo
O objetivo deste trabalho foi avaliar a deposição de serapilheira e nutrientes sob diferentes métodos de restauração florestal em áreas degradadas pela mineração de bauxita no município de Paragominas, Pará. Os métodos utilizados para restauração florestal foram: plantio de mudas arbóreas (PM) e indução da regeneração natural (RN), além disso, avaliou-se um fragmento florestal (FF) como referência. Foram instalados 30 coletores de serapilheira de $0,25 \mathrm{~m}^{2}$ de abertura em cada ambiente. A serapilheira foi coletada, seca em estufa e quantificada mensalmente de agosto de 2014 a setembro de 2015. Posteriormente, todo material foi analisado quimicamente para obtenção das concentrações dos elementos $\mathrm{N}, \mathrm{P}, \mathrm{K}, \mathrm{Ca}, \mathrm{Mg}, \mathrm{S}, \mathrm{Fe}$, $\mathrm{Mn}, \mathrm{Cu}$ e $\mathrm{Zn}$. Por meio dos valores das concentrações, foram obtidos os valores dos conteúdos. A deposição de serapilheira foi de $6,61 \pm 0,53,10,75 \pm 0,86$ e $11,83 \pm 1,00 \mathrm{Mg} \mathrm{ha}^{-1} \mathrm{ano}^{-1}$ no PM, FF e RN respectivamente, diferindo estatisticamente entre si $(p=0,0025)$. As concentrações de $\mathrm{N}, \mathrm{P}, \mathrm{K}, \mathrm{Mg}$ e Ca foram superiores na área de PM na maioria dos meses de avaliação devido às adubações de pré-plantio. $\mathrm{O} S$ foi o elemento que mais variou entre as áreas de estudo. Com relação aos micronutrientes, o $\mathrm{Cu}, \mathrm{Fe}, \mathrm{Zn}$ e $\mathrm{Mn}$ tiveram as menores concentrações no FF e o $\mathrm{Mn}$ as maiores concentrações no ecossistema RN. Os conteúdos de $\mathrm{N}$ e $\mathrm{K}$ da serapilheira foram superiores nos ecossistemas RN, não diferindo do FF. A RN foi o método mais eficiente de restauração. A serapilheira é um bom indicador de restauração de áreas degradadas.
\end{abstract}

Palavras-chave: Indução da regeneração, ciclagem de nutrientes, monitoramento florestal.

\begin{abstract}
Deposition of litterfall and nutrients in areas of mining submitted to forest restoration methods in Paragominas, Pará. The objective of this study was to evaluate the deposition of litterfall and nutrients under different forest restoration methods in areas degraded by bauxite mining in the municipality of Paragominas, state of Pará, Brazil. The methods used for forest restoration were: planting of tree seedlings (PM) and induction of natural regeneration ( $\mathrm{RN}$ ); in addition, a forest fragment (FF) was evaluated as reference. 30 litter collectors with $0.25 \mathrm{~m}^{2}$ opening were installed in each room. The material was chemically analyzed to obtain the concentrations of the chemical elements $\mathrm{N}, \mathrm{P}, \mathrm{K}, \mathrm{Ca}, \mathrm{Mg}, \mathrm{S}, \mathrm{Fe}, \mathrm{Mn}, \mathrm{Cu}$, and $\mathrm{Zn}$. The values of the contents were obtained by means of the concentration values. The litterfall deposition was $6.61 \pm 0.53$, $10.75 \pm 0.86$ and $11.83 \pm 1.00 \mathrm{Mg}$ ha- $1 \mathrm{yr}^{-1}$ for PM, FF and $\mathrm{RN}$ respectively, differing statistically from each other ( $\mathrm{p}=0.0025$ ). The concentration of $\mathrm{N}, \mathrm{P}, \mathrm{K}, \mathrm{Mg}$ and $\mathrm{Ca}$ was higher in the PM area in most assessment months due to planting fertilization. The $\mathrm{S}$ was the element that varied the most among the study areas. Regarding micronutrients, $\mathrm{Cu}, \mathrm{Fe}, \mathrm{Zn}$ and $\mathrm{Mn}$ had the lowest concentration in FF, and $\mathrm{Mn}$ had the highest concentrations in the RN ecosystem. The contents of $\mathrm{N}$ and $\mathrm{K}$ of the litter were higher in the RN ecosystems, not differing from the FF. The RN was the most efficient method of restoration. Litterfall is a good indicator of restoration of degraded areas.

Keywords: Induction of regeneration, nutrient cycling, forest monitoring.
\end{abstract}

\section{INTRODUÇÃO}

A mineração contribui significativamente para a economia brasileira, gerando, aproximadamente, 2,2 milhões de empregos diretos (LIMA; NEVES, 2014). Nesse cenário, o estado do Pará se destaca por ser o principal produtor de bauxita do país, cerca de $90 \%$ da produção nacional (LIMA; NEVES, 2014). Porém, a atividade minerária causa sérios impactos ambientais, principalmente pela retirada da vegetação e das camadas superficiais e subsuperficiais do solo, modificando a paisagem como um todo do ecossistema (JESUS et al., 2016).

FLORESTA, Curitiba, PR, v. 48, n. 1, p. 37-48, jan./marc. 2018.

Martins.W. B. R. et al.

ISSN eletrônico 1982-4688

DOI: $10.5380 /$ rf.v48 i1.49288 
Como parte dos esforços para a manutenção de processos naturais de restauração, há o plantio de mudas de espécies nativas arbóreas. Este tem sido um dos métodos mais utilizados no processo de restauração de áreas degradadas pela mineração. Porém, esse método é considerado oneroso. Além disso, a mortalidade das mudas em áreas mineradas tem sido um problema, sobretudo nos primeiros anos, e não garante o sucesso do projeto muitas vezes.

A indução da regeneração natural por meio da devolução do solo superficial (topsoil), retirado após a supressão da vegetação, também tem sido utilizada para restaurar áreas degradadas, pois, de acordo com Guimarães et al. (2014), o banco de sementes de ecossistemas florestais é constituído por uma grande densidade de indivíduos e riqueza de espécies que auxiliam no reestabelecimento da vegetação local.

No processo de restauração florestal, independentemente do método utilizado, torna-se necessária a execução de monitoramentos periódicos para avaliação de indicadores ou variáveis que demonstrem o desenvolvimento da restauração, sendo possível, com esses resultados, avaliar se a evolução do processo de restauração está seguindo uma trajetória planejada ou se é preciso readequá-lo. Para isso, torna-se necessário avaliar um ecossistema de referência que esteja nas proximidades das áreas em restauração concomitantemente, pois remanescentes ou fragmentos florestais distantes comprometem a regeneração natural por meio da dispersão de sementes, realizada pela fauna silvestre (KAUANO et al., 2013).

Um dos indicadores, ou variáveis, que podem ser utilizados para monitorar o processo de restauração florestal é a serapilheira, formada pela deposição de folhas, galhos, material reprodutivo e carcaças de animais (RODRIGUES, 2010). Essa variável contribui para a formação e manutenção da fertilidade dos solos através do processo de decomposição, sobretudo nas florestas amazônicas, que são consideradas pouco férteis naturalmente (QUESADA et al., 2011).

Alguns estudos foram desenvolvidos com serapilheira em áreas em processo de restauração florestal (GOMES et al., 2011; WANG'ONDU et al., 2014; SOUZA et al., 2016). Isso mostra que o aumento da deposição de serapilheira e nutrientes contribui positivamente para acelerar o desenvolvimento do ecossistema (MACHADO et al., 2015; LONDE et al., 2016). Além disso, a camada de serapilheira em ecossistemas em início de restauração florestal impede ou minimiza os processos erosivos (VILLA et al., 2016), que são comuns em áreas mineradas, principalmente na região amazônica, onde o período chuvoso é intenso.

A ciclagem de nutrientes por meio da decomposição da serapilheira varia em função das características físicas e químicas do solo, índice pluviométrico, radiação solar e composição florística. Destacam-se as características das espécies de absorver e redistribuir os nutrientes (VITOUSEK, 1982; MACHADO et al., 2016), podendo variar, também, de acordo com o espaçamento de plantio das mudas, idade e estágio de desenvolvimento sucessional da floresta em processo de restauração (VILLA et al., 2016). O conhecimento da concentração e do conteúdo dos nutrientes advindos da serapilheira é importante, pois a baixa qualidade nutricional do material vegetal influencia a fertilidade do solo de maneira negativa e a longo prazo, prejudicando a restauração (PAUDEL et al., 2015).

Neste estudo, foram avaliados diferentes métodos de restauração relacionados à capacidade de deposição e qualidade nutricional da serapilheira para recuperação de áreas degradadas, de modo a responder a seguinte pergunta: qual é o melhor método de restauração florestal para áreas degradadas pela mineração de bauxita: plantio de mudas de espécies arbóreas ou a indução da regeneração natural? A hipótese utilizada foi a de que a área de indução de regeneração natural apresenta maior produção de serapilheira e nutrientes em decorrência do rápido crescimento inicial de espécies florestais arbóreas. Assim, o objetivo deste trabalho foi avaliar a deposição de nutrientes da serapilheira em áreas de mineração de bauxita sob dois métodos de restauração florestal e uma área controle no município de Paragominas, Pará.

\section{MATERIAL E MÉTODOS}

\section{Área de estudo}

O estudo foi desenvolvido na empresa Mineração Paragominas S.A. do grupo Hydro, localizada no nordeste do Pará, no Platô Miltônia 3 (3¹5'38"S e 4743’28’'O) a uma altitude de 150 m, distante 70 km da sede municipal de Paragominas. O clima da região é do tipo Awi, segundo a classificação de Köppen, caracterizado como quente e úmido com estações de chuva e seca bem definidas, com temperatura média em torno de $26,7{ }^{\circ} \mathrm{C}$. Os totais anuais de precipitação da região variam de 857,8 a $2.787 \mathrm{~mm}$, com o período mais chuvoso sendo de janeiro a maio (Figura 1). 


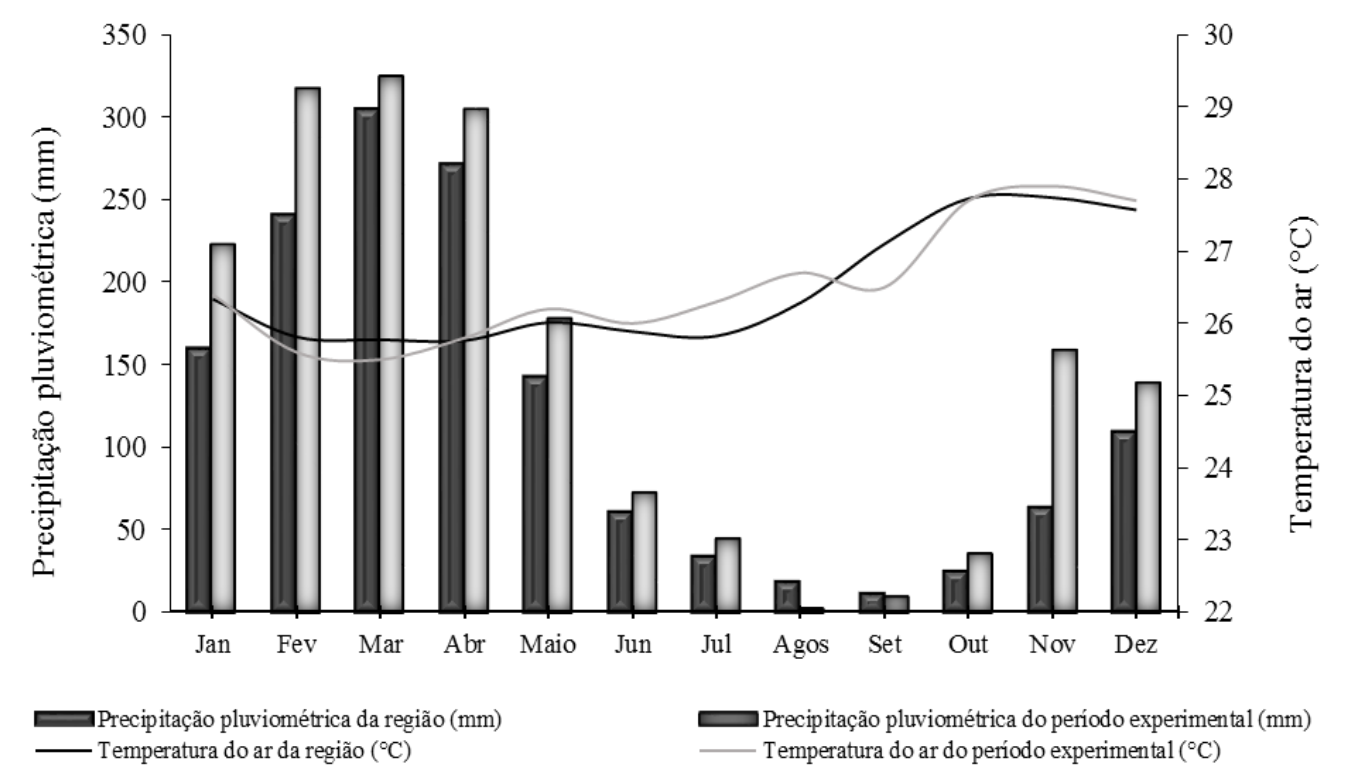

Figura 1. Média mensal da precipitação pluviométrica e temperatura média do ar do município de Paragominas, Pará, dos últimos sete anos (2008-2014) e do período experimental da área de mineração (setembro de 2014 a outubro de 2015). Fonte: Hydro (2015); INMET (2015).

Figure 1. Average monthly rainfall and average air temperature of the municipality of Paragominas, state of Pará, Brazil, of the last seven years (2008-2014) and the experimental period of the mining area (September, 2014 to October, 2015). Source: Hydro (2015); INMET (2015).

Os solos predominantes do município são classificados como Latossolo Amarelo, Argissolo Amarelo, Plintossolo, Gleissolo e Neossolo, considerados de baixa fertilidade natural. Apresentam baixas concentrações de cálcio, magnésio, potássio, fósforo e nitrogênio, além da alta saturação de alumínio (RODRIGUES et al., 2003).

\section{Caracterização dos ecossistemas de estudo}

Foram selecionadas três áreas para coleta da serapilheira: duas em processo de restauração florestal (A1 - plantio de mudas arbóreas nativas, A2 - indução da regeneração natural); e uma área de referência (A3 fragmento florestal), localizada a $300 \mathrm{~m}$ de distância das duas áreas em restauração. Nas áreas selecionadas para restauração, foram realizadas as seguintes atividades: supressão da vegetação florestal, extração do minério, fechamento da mina, reconformação topográfica e espalhamento do topsoil, o qual foi retirado a $20 \mathrm{~cm}$ de profundidade e ficou armazenado em leiras durante um ano.

A1 - Plantio de mudas arbóreas nativas (PM)

O plantio foi realizado em uma área de 20 ha, com a realização da subsolagem e aplicação de fosfato natural reativo (33\% de $\mathrm{P}_{2} \mathrm{O}_{5}$ total e $10 \%$ de $\mathrm{P}_{2} \mathrm{O}_{5}$ solúvel em ácido cítrico a $2 \%$ ) no fundo do sulco. Além disso, foram aplicados $800 \mathrm{~kg} \mathrm{ha}^{-1}$ de calcário dolomítico em área total e $200 \mathrm{~g}$ por cova de adubo NPK (06-30-06) mais micronutrientes $(0,5 \% \mathrm{~B}, 0,5 \% \mathrm{Cu}, 0,5 \% \mathrm{Zn})$ e $2,5 \mathrm{~kg}$ de composto orgânico por cova, constituído de terra vegetal, caroço de açaí carbonizado, palha de arroz carbonizado, cama de frango curtido e triturado e esterco de ovino curtido e triturado na proporção de $20 \%$ de cada componente. O plantio das mudas foi realizado em maio de 2009 em covas de $0,30 \times 0,30 \times 0,30 \mathrm{~m}$, espaçadas cerca de 3 x $3 \mathrm{~m}$. Foram utilizadas, inicialmente, 105 espécies de diferentes grupos ecológicos (38\% pioneiras, 28\% secundárias iniciais e 34\% espécies secundárias tardias). Para a manutenção inicial do plantio, realizou-se o controle de formigas cortadeiras com isca formicida granulada e duas capinas manuais em área total durante o primeiro ano.

O plantio tinha sete anos de idade, com altura média do estrato superior de, aproximadamente, 5,0 m e 106 espécies arbóreas distribuídas em 23 famílias. As dez espécies com os maiores Índices de Valor de Importância (IVI) relativos (\%) foram: Chloroleucon acacioides (Ducke) Barneby \& J.W. Grimes $(4,00)$,

FLORESTA, Curitiba, PR, v. 48, n. 1, p. 37-48, jan./marc. 2018.

Martins.W. B. R. et al.

ISSN eletrônico 1982-4688

DOI: $10.5380 /$ rf.v48 i1.49288 
Libidibia férrea (Mart.) L.P. Queiroz (3,69), Inga alba (Sw.) Willd. (3,67), Mimosa schomburgkii Benth. $(3,66)$, Ceiba pentandra (L) Gaertn. (3,66), Genipa americana L. (3,35), Inga laurina (Sw.) Willd (3,31), Croton matourensis Aubl. $(2,90)$, Swietenia macrophylla King $(2,63)$ e Byrsonima crassifolia (L.) Kunth $(2,43)$.

\section{A2 - Indução da Regeneração Natural (RN)}

Os procedimentos realizados no ecossistema de indução de regeneração natural consistiam apenas na reconformação do terreno e no espalhamento do topsoil em área total, com posterior isolamento da área. $\mathrm{O}$ ecossistema tinha área de 20 ha, 7 anos de idade e 26 espécies arbóreas com altura média do estrato superior a 3,5 m, distribuídas em 12 famílias com hábito predominantemente arbóreo. As espécies predominantes com maior IVI relativo (\%) foram: Croton matourensis Aubl. $(18,65)$, Vismia guianensis (Aubl.) Choisy $(14,66)$, Cecropia sp. (14,08), Byrsonima crispa A. Juss. (5,44), Solanum sp. (5,28), Casearia grandiflora Cambess. $(3,40)$, Cheiloclinium sp. $(3,32)$, Guatteria poeppigiana Mart. $(3,19)$, Croton ascendens Secco \& N.A. Rosa $(3,10)$ e Casearia arborea (Rich.) Urb. $(2,68)$.

\section{A3 - Fragmento Florestal (FF)}

Foi selecionada uma área de aproximadamente 20 ha de um fragmento florestal classificado como floresta secundária de terra firme, tendo último registro de exploração madeireira convencional de $2003 \mathrm{e}$ apresentando, atualmente, 11 anos pós-exploração. Esse ecossistema tem 51 espécies arbóreas com altura média do estrato superior a 7,5 m, distribuídas em 27 famílias, destacando-se, com maior IVI (\%), as espécies: Croton matourensis Aubl. (8,12), Tapirira guianensis Aubl. (7,80), Inga alba (Sw) Willd. (7,54), Chrysophyllum prieurii A.DC. (7,08), Inga thibaudiana DC. $(5,62)$, Guatteria poeppigiana Mart. $(5,50)$, Cordia scabrifolia A.DC. (5,02), Vismia guianensis (Aubl.) Choisy $(4,26)$, Eschweilera coriacea (DC.) S.A. Mori $(4,06)$ e Myrcia splendens (Sw.) DC. (3,31).

\section{Coleta e análise dos dados}

Para coleta de serapilheira, foram instalados coletores confeccionados de madeira e sombrite, com área de coleta de $0,25 \mathrm{~m}^{2}(0,5 \mathrm{~m} \times 0,5 \mathrm{~m})$ e altura de $0,1 \mathrm{~m}$, alocados $0,2 \mathrm{~m}$ acima da superfície do solo para reduzir o contato. Foram instalados 90 coletores em agosto de 2014 distribuídos sistematicamente, sendo 30 coletores em cada ecossistema de pesquisa distantes $30 \mathrm{~m}$ entre si. As coletas foram realizadas mensalmente durante os meses de agosto de 2014 a setembro de 2015. Após a retirada da serapilheira, o material foi transportado em sacos plásticos para o laboratório de solos e nutrição de plantas da Universidade Federal Rural da Amazônia (UFRA), onde foi colocado para secagem em estufa a $70^{\circ} \mathrm{C}$ por 72 horas. Posteriormente, o material foi pesado em balança analítica de precisão de $0,01 \mathrm{~g}$ e triturado em um moinho de lâminas do tipo Willey com peneira de $0,85 \mathrm{~mm}$ de abertura. As amostras coletadas mensalmente foram homogeneizadas para obtenção de quatro amostras compostas de cada área de estudo, totalizando 108 análises ao final de 12 meses.

As concentrações dos macronutrientes Nitrogênio (N), Fósforo (P), Potássio (K), Cálcio (Ca), Magnésio $(\mathrm{Mg})$, Enxofre (S) e dos micronutrientes Ferro (Fe), Manganês $(\mathrm{Mn})$, Cobre $(\mathrm{Cu})$ e Zinco $(\mathrm{Zn})$ foram analisadas no Laboratório do Museu Emílio Goeldi, no município de Belém, Pará, seguindo o método descrito por Malavolta et al. (1997). O conteúdo de nutrientes, que se refere à quantidade total de cada elemento que retornará para o solo, foi estimado por meio da multiplicação da concentração média mensal de cada nutriente pela respectiva produção de serapilheira de cada ecossistema. O conteúdo total anual foi calculado pela soma mensal (Vitousek, 1982).

Os resultados de massa seca da serapilheira, das concentrações e dos conteúdos de cada nutriente foram submetidos ao teste de normalidade de D'Agostino-Pearson e homocedasticidade de variância de Bartlett. Posteriormente, foi realizada uma análise de variância (ANOVA) para constatação mensal de diferenças estatísticas entre os ecossistemas para as três variáveis e outra análise de variância para a deposição de serapilheira e conteúdo de cada elemento. Quando houve diferenças significativas entre os dados, as médias foram comparadas pelo teste Tukey, ao nível de 5\% de probabilidade de erro. As análises foram realizadas no programa Statistica 9.0 e a confecção das figuras no software SigmaPlot 10.0.

\section{RESULTADOS}

\section{Deposição de serapilheira}

A deposição de serapilheira foi de $6,61 \pm 0,53,10,75 \pm 0,86$ e $11,83 \pm 1,00 \mathrm{Mg} \mathrm{ha}^{-1}$ ano $^{-1}$ para PM, RN e FF, respectivamente, diferindo estatisticamente entre si $\left(\mathrm{F}_{2 ; 1044}=158,63, p<0,001\right)$. Os maiores valores mensais, obtidos pelo somatório dos valores dos três ecossistemas, foram registrados em agosto, setembro e julho, com 3,77; 3,59; e 3,51 Mg ha', nesta ordem (Figura 2). 


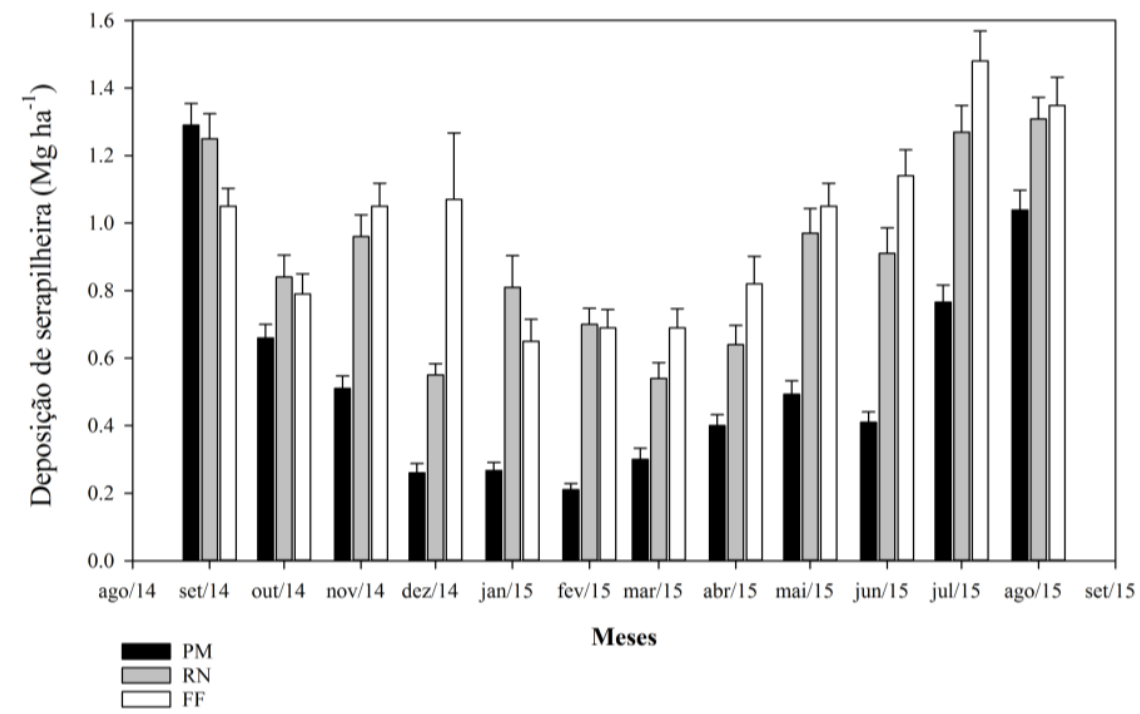

Figura 2. Média $( \pm \mathrm{Sd})$ mensal de serapilheira em área de restauração florestal com método de plantio de mudas $(\mathrm{PM})$ e regeneração natural (RN) e um fragmento florestal utilizado como testemunha (FF).

Figure 2. Monthly $( \pm \mathrm{Sd})$ litter deposition in forest restoration area with seedlings planting method (PS) and natural regeneration (NR) and a forest fragment used as control (FF).

\section{Concentrações de nutrientes da serapilheira}

De modo geral, N, K, Ca e S foram os elementos com as maiores concentrações entre os meses de julho e novembro nas três áreas de estudo (Figura 3). Em contrapartida, a concentração de $\mathrm{P}$ foi maior no período de dezembro a março para os três ambientes (Figura 3D). 

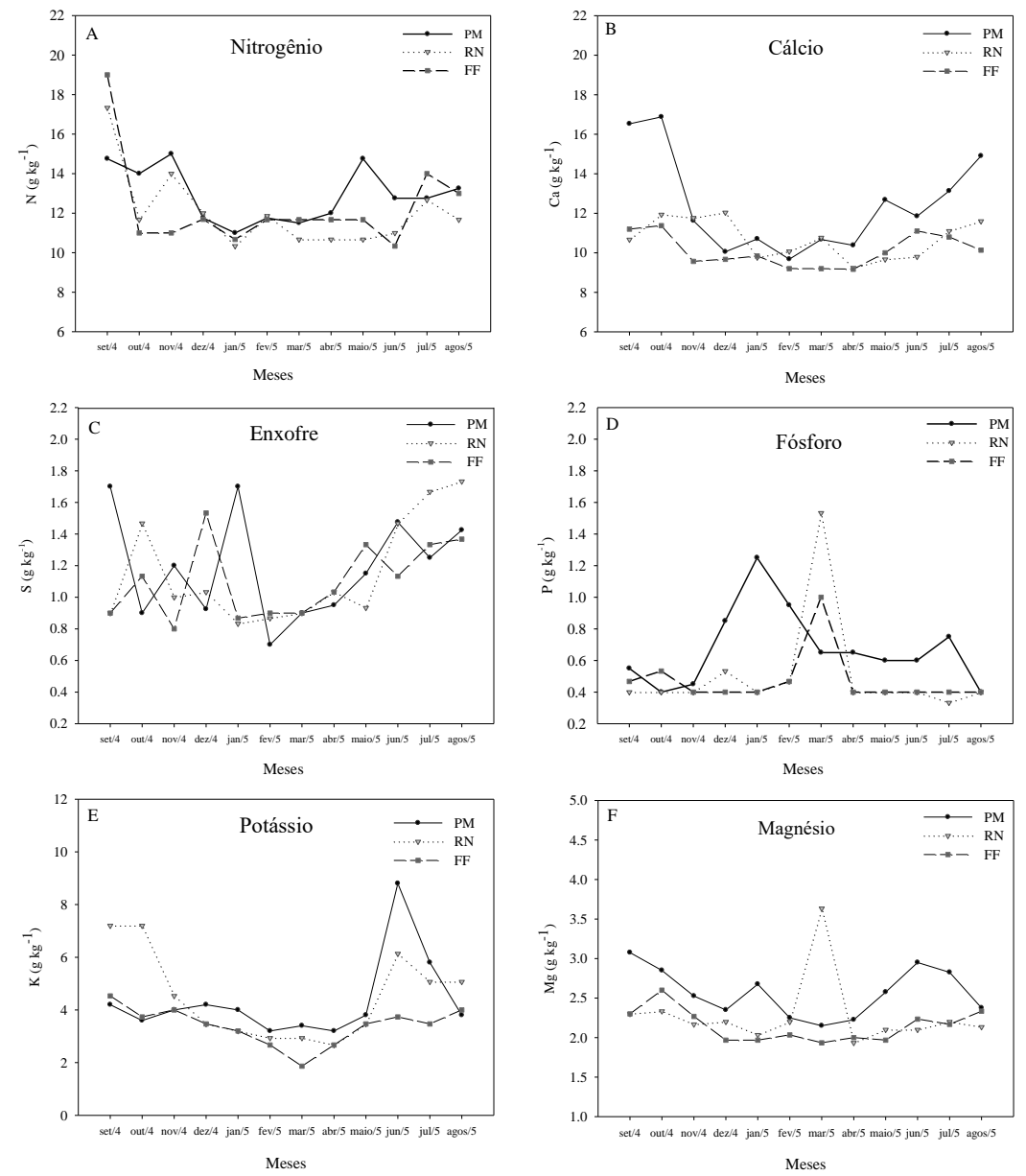

Figura 3. Fluxo de concentração média de macronutrientes de serapilheira em área de restauração florestal com método de plantio de mudas (PM), regeneração natural (RN) e um fragmento florestal utilizado como testemunha (FF). A: Nitrogênio; B: Cálcio; C: Enxofre; D: Fósforo; E: Potássio; F: Magnésio.

Figure 3. Flow of average concentration of litter macronutrients in forest restoration area with seedlings planting method (PS), natural regeneration (NR) and a forest fragment used as control (FF). A: Nitrogen; B: Calcium; C: Sulphur; D: Phosphorus; E: Potassium; F: Magnesium.

As concentrações de $\mathrm{N}, \mathrm{P}, \mathrm{K}, \mathrm{Mg}$ e Ca foram superiores na área de plantio de mudas arbóreas na maioria dos meses de avaliação $(p<0,05)$ (Figura 3 ). $\mathbf{S}$ foi o elemento que mais variou, sendo cinco dos doze meses superiores no método de indução da regeneração natural [outubro $\left(\mathrm{F}_{2 ; 9}=11,57, p=0,003\right)$; fevereiro $\left(\mathrm{F}_{2 ; 9}=6,91, p=0,019\right)$; junho $\left(\mathrm{F}_{2 ; 9}=6,91, p=0,015\right)$; julho $\left(\mathrm{F}_{2 ; 9}=6,86, p=0,004\right)$; e agosto $\left.\left(\mathrm{F}_{2 ; 9}=8,62, p=0,008\right)\right]$. Quatro dos doze meses apresentaram médias superiores no método de plantio de mudas arbóreas [setembro $\left(\mathrm{F}_{2 ; 9}=13,71, p=0,002\right)$; novembro $\left(\mathrm{F}_{2 ; 9}=12,00, p=0,003\right)$; janeiro $\left(\mathrm{F}_{2 ; 9}=14,15, p=0,002\right)$; e junho $\left.\left(\mathrm{F}_{2 ; 9}=6,86, p=0,015\right)\right]$. Não houve tendência clara entre os tratamentos.

No que se refere aos micronutrientes, $\mathrm{Cu}, \mathrm{Fe}, \mathrm{Zn}$ e $\mathrm{Mn}$ foram menores no $\mathrm{FF}$, com baixa variação ao longo dos meses (Figura 4). Mn foi o elemento que apresentou maiores concentrações no ecossistema RN durante os doze meses. Quando analisado o aumento desses elementos durante um ano no PM e RN, observou-se que ocorreram picos de crescimento em fevereiro e março para $\mathrm{Zn}$ e de dezembro a fevereiro para $\mathrm{Cu}$ (Figura $4 \mathrm{~A}$ e D). 

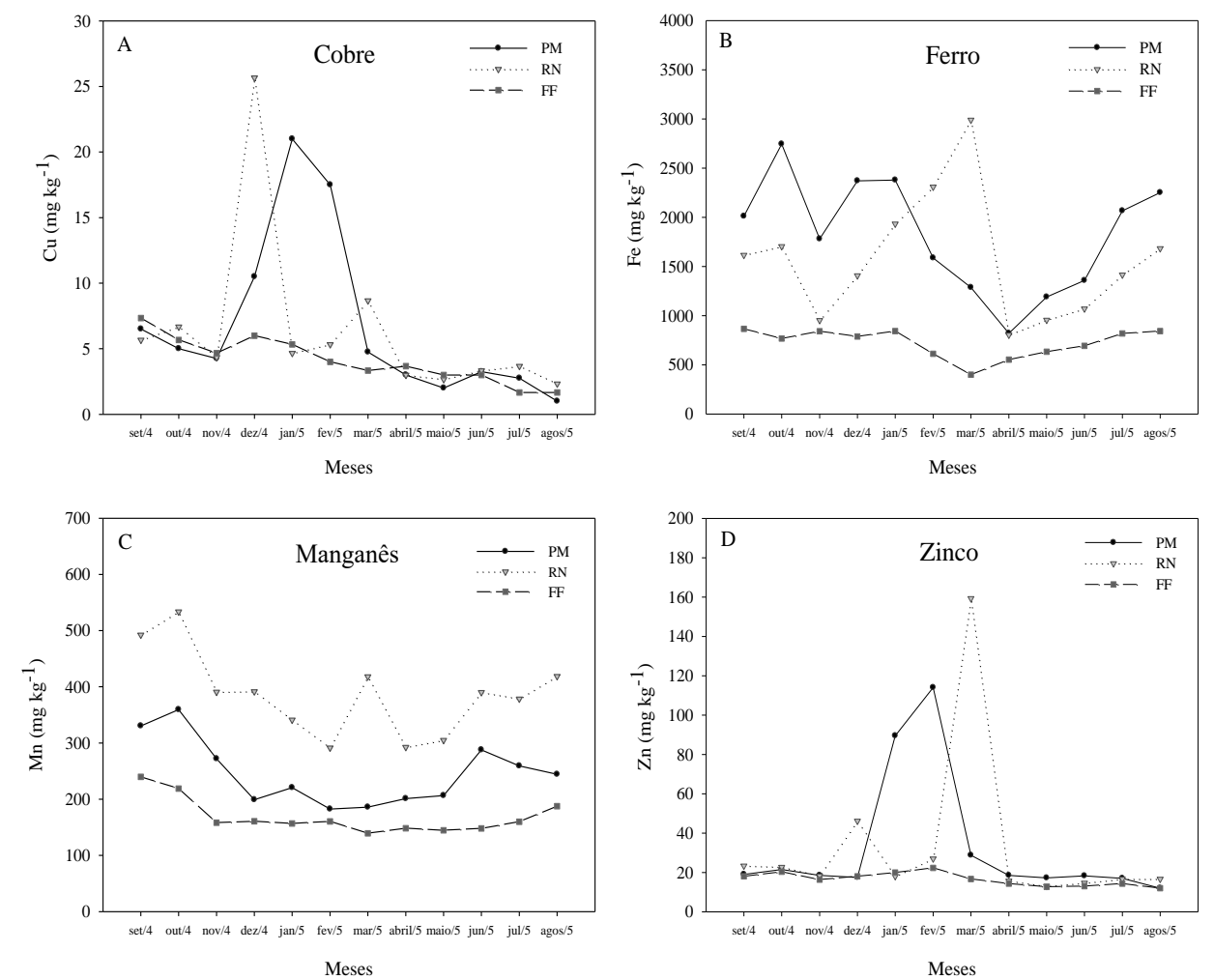

Figura 4. Fluxo da concentração média de micronutrientes da serapilheira em área de restauração florestal com método de plantio de mudas (PM), regeneração natural (RN) e um fragmento florestal utilizado como testemunha (FF). A: Cobre; B: Ferro; C: Manganês; D: Zinco.

Figure 4. Flow of average concentration of litter micronutrients in forest restoration area with seedlings planting method (PS), natural regeneration (NR) and a forest fragment used as control (FF). A: Copper; B: Iron; C: Manganese; D: Zinc.

\section{Conteúdo de nutrientes da serapilheira}

Os conteúdos de $\mathrm{N}$ e $\mathrm{K}$ da serapilheira foram maiores nos ecossistemas $\mathrm{RN}$, não diferindo de $\mathrm{FF}$ (Tabela 1). Apesar de não ter sido constatada diferença significativa, $\mathrm{P}, \mathrm{Ca}, \mathrm{Mg}$ e $\mathrm{S}$ foram superiores numericamente em FF, seguido de RN (Tabela 1).

Tabela 1. Conteúdo de nutrientes da serapilheira em área de restauração florestal com método de plantio de mudas (PM) e regeneração natural (RN) e um fragmento florestal utilizado como testemunha (FF).

Table 1. Litter nutrient content in forest restoration area with seedlings planting method (PS) and natural regeneration (NR) and a forest fragment used as control (FF).

\begin{tabular}{|c|c|c|c|c|c|}
\hline \multirow{2}{*}{ Nutrientes } & \multicolumn{5}{|c|}{ Conteúdo $\left(\mathrm{Kg} \mathrm{ha}^{-1} \mathrm{ano}^{-1}\right)$} \\
\hline & & PM & RN & FF & $p$-valor \\
\hline $\mathrm{N}$ & $\begin{array}{l}\text { Média mensal } \\
\text { Total }\end{array}$ & $\begin{array}{c}7,393( \pm 1,44) b \\
88,713\end{array}$ & $\begin{array}{c}11,041( \pm 1,36) \mathrm{a} \\
132,492\end{array}$ & $\begin{array}{c}12,300( \pm 1,36) \text { a } \\
147,598\end{array}$ & 0,0455 \\
\hline $\mathrm{P}$ & $\begin{array}{l}\text { Média mensal } \\
\text { Total }\end{array}$ & $\begin{array}{c}0,329( \pm 0,05) \\
3,949 \\
\end{array}$ & $\begin{array}{c}0,412( \pm 0,04) \\
4,957 \\
\end{array}$ & $\begin{array}{c}0,447( \pm 0,03) \\
5,368 \\
\end{array}$ & 0,1440 \\
\hline K & $\begin{array}{l}\text { Média mensal } \\
\text { Total }\end{array}$ & $\begin{array}{c}2,406( \pm 0,45) b \\
28,866\end{array}$ & $\begin{array}{c}4,272( \pm 0,70) \text { a } \\
51,259 \\
\end{array}$ & $\begin{array}{c}3,453( \pm 0,40) \mathrm{a} \\
41,441 \\
\end{array}$ & 0,0410 \\
\hline $\mathrm{Ca}$ & $\begin{array}{l}\text { Média mensal } \\
\text { Total }\end{array}$ & $\begin{array}{c}7,495( \pm 1,72) \\
89,941 \\
\end{array}$ & $\begin{array}{c}9,629( \pm 0,93) \\
115,543 \\
\end{array}$ & $\begin{array}{c}10,046( \pm 0,90) \\
120,552 \\
\end{array}$ & 0,3110 \\
\hline $\mathrm{Mg}$ & $\begin{array}{l}\text { Média mensal } \\
\text { Total }\end{array}$ & $\begin{array}{c}1,466( \pm 0,29) \\
17,593 \\
\end{array}$ & $\begin{array}{c}2,004( \pm 0,16) \\
24,047 \\
\end{array}$ & $\begin{array}{c}2,131( \pm 0,19) \\
25,577 \\
\end{array}$ & 0,0960 \\
\hline$S$ & $\begin{array}{l}\text { Média mensal } \\
\text { Total }\end{array}$ & $\begin{array}{c}0,709( \pm 0,17) \\
8,508\end{array}$ & $\begin{array}{c}1,078( \pm 0,17) \\
12,941\end{array}$ & $\begin{array}{c}1,124( \pm 0,14) \\
13,483\end{array}$ & 0,1478 \\
\hline
\end{tabular}

FLORESTA, Curitiba, PR, v. 48, n. 1, p. 37-48, jan./marc. 2018.

Martins.W. B. R. et al.

ISSN eletrônico 1982-4688

DOI: $10.5380 /$ rf.v48 i1.49288 


\begin{tabular}{|c|c|c|c|c|c|}
\hline $\mathrm{Cu}$ & $\begin{array}{l}\text { Média mensal } \\
\text { Total }\end{array}$ & $\begin{array}{c}0,003( \pm 0,00) \\
0,034\end{array}$ & $\begin{array}{c}0,005( \pm 0,00) \\
0,058\end{array}$ & $\begin{array}{c}0,004( \pm 0,00) \\
0,046\end{array}$ & 0,0959 \\
\hline $\mathrm{Fe}$ & $\begin{array}{l}\text { Média mensal } \\
\text { Total }\end{array}$ & $\begin{array}{c}1,057( \pm 0,23) \mathrm{ab} \\
12,689\end{array}$ & $\begin{array}{c}1,363( \pm 0,15) \mathrm{a} \\
16,354\end{array}$ & $\begin{array}{c}0,729( \pm 0,08) b \\
8,745\end{array}$ & 0,0320 \\
\hline $\mathrm{Mn}$ & $\begin{array}{l}\text { Média mensal } \\
\text { Total }\end{array}$ & $\begin{array}{c}0,147( \pm 0,03) b \\
1,761\end{array}$ & $\begin{array}{c}0,352( \pm 0,04) \mathrm{a} \\
4,228\end{array}$ & $\begin{array}{c}0,167( \pm 0,02) b \\
2,004\end{array}$ & 0,0002 \\
\hline $\mathrm{Zn}$ & $\begin{array}{l}\text { Média mensal } \\
\text { Total }\end{array}$ & $\begin{array}{c}0,013( \pm 0,00) \\
0,158\end{array}$ & $\begin{array}{c}0,024( \pm 0,01) \\
0,290\end{array}$ & $\begin{array}{c}0,016( \pm 0,00) \\
0,189\end{array}$ & 0,0894 \\
\hline
\end{tabular}

Quanto aos micronutrientes, Fe e Mn diferiram estatisticamente entre as áreas de estudo. Fe apresentou as maiores médias no PM e RN, não havendo diferença entre si. Mn foi superior na regeneração natural com diferença altamente significante em relação aos demais ecossistemas (Tabela 1).

\section{DISCUSSÃO}

\section{Deposição de serapilheira}

Os valores de deposição anual de serapilheira das áreas analisadas ficaram próximos aos encontrados em outros estudos realizados na Amazônia em florestas de terra firme. De acordo com Zhang et al. (2014), a deposição de serapilheira para florestas tropicais se encontra em torno de 7,0 $\mathrm{Mg} \mathrm{ha}^{1}{ }^{\text {ano }}{ }^{-1}$, valor compatível com os resultados do presente estudo.

Nos meses de maior deposição (setembro a novembro de 2014 e junho a agosto de 2015), foram constatados maiores valores de temperatura e baixa precipitação pluviométrica, o que ocasionou abscisão foliar, uma estratégia das plantas para controle de perda de água por transpiração (SANCHES et al., 2008; MACHADO et al., 2015). No entanto, Kim et al. (2012) mencionam que a maior produção de serapilheira em períodos mais quentes na Amazônia está associada à renovação foliar das árvores para melhor aproveitamento da radiação fotossinteticamente ativa, mesmo diante de pouco recurso hídrico disponível no solo.

As maiores médias de serapilheira observadas nas áreas de RN e FF estão relacionadas, possivelmente, ao domínio de espécies pioneiras de rápido crescimento, como Croton matourensis, Vismia guianensis, Tapirira guianensis e Inga alba. Essas espécies desenvolvem-se rapidamente em áreas antropizadas e investem mais energia na produção de folhas do que de madeira em decorrência da competição por recursos, principalmente por luz (GUARIGUATA; OSTERTAG, 2001).

\section{Concentrações de nutrientes da serapilheira}

As maiores concentrações de $\mathrm{N}, \mathrm{K}, \mathrm{Ca}$ e $\mathrm{S}$ entre os meses de julho e novembro são explicadas pelo período considerado menos chuvoso na área de estudo, com precipitação média de $51 \mathrm{~mm}$ mês $\mathrm{s}^{-1}$, segundo dados da estação meteorológica do Mineração Paragominas (MPSA). Já o resultado inverso de P, com elevada concentração nos meses considerados chuvosos (média $251 \mathrm{~mm}$ mês ${ }^{-1}$ ), demostra que as primeiras chuvas causam elevadas transferências deste elemento contido na serapilheira para o perfil do solo (SELLE, 2007).

A adubação inicial na área de PM pode ter sido o principal fator que contribuiu para as maiores concentrações mensais dos macronutrientes $\mathrm{N}, \mathrm{P}, \mathrm{K}, \mathrm{Ca}$ e $\mathrm{Mg}$ no ecossistema PM. Isso demostra a eficiência da aplicação de fertilizantes pré-plantio, dentre eles a calagem, adubação NPK e fosfatagem natural durante a subsolagem.

Machado et al. (2016) relatam que espécies do grupo ecológico das pioneiras apresentam maiores concentrações de $\mathrm{N}$ em relação às espécies não pioneiras. O predomínio de espécies da família fabaceae no plantio de mudas (seis das dez espécies com maior VI) fez com que a concentração de $\mathrm{N}$ fosse maior, sobretudo nas folhas, principal componente da serapilheira (Machado et al., 2016). Segundo Malavolta et al. (1997), N é o mineral requerido em maior quantidade pelas plantas. Por essa razão, sua ciclagem por meio da decomposição da serapilheira torna-se fundamental para o desenvolvimento inicial de ecossistemas em processo de restauração florestal.

Já Ca é um dos principais componentes da estrutura celular vegetal, sendo um dos últimos elementos a ser liberado para o solo pelo processo de decomposição (GODINHO et al., 2014). O retorno de Ca para o solo é importante como regulador do $\mathrm{pH}$ da superfície do próprio solo, proporcionando condições adequadas para o crescimento inicial das plantas, principalmente em áreas fortemente antropizadas, como é o caso da mineração.

$\mathrm{P}$, depois da deficiência hídrica, é considerado o elemento que mais limita o crescimento das árvores nos ecossistemas florestais tropicais (VITOUSEK, 1982). Por causa disso, de acordo com Machado et al. (2016), ele é retranslocado para outras partes da planta no período menos chuvoso antes da abscisão foliar, uma estratégia de otimização eficaz no uso do elemento nos processos metabólicos do vegetal. Neste sentido, a maior concentração de P nos três ecossistemas foi no período mais chuvoso, momento em que as perdas de fósforo são 
compensadas pala absorção do elemento na solução do solo. Com isso, a concentração mensal de P foi superior em sete dos doze meses de avaliação no ecossistema de plantio de mudas, o que está, de fato, relacionado à aplicação de fosfato, sobretudo na formulação NPK.

A baixa contribuição da concentração dos micronutrientes da serapilheira e a ocorrência de possíveis contaminações foram os principais fatores responsáveis pela indiferença dos elementos $\mathrm{Cu}$ e $\mathrm{Zn}$ durante todos os meses de avaliação entre as áreas de estudo. Já os maiores valores mensais de Fe e Mn nas áreas de RN e PM em relação ao FF podem estar relacionados às modificações edáficas recentes e às misturas de horizontes no momento da recomposição topográfica para o início da restauração florestal.

\section{Conteúdo de nutrientes da serapilheira}

O maior conteúdo dos nutrientes nas áreas de regeneração natural em relação ao método de plantio de mudas arbóreas está relacionado, provavelmente, à maior produção anual de serapilheira. Apesar disso, não ocorreu diferença entre os ecossistemas quanto ao conteúdo de $\mathrm{P} \mathrm{Ca}, \mathrm{Mg}$ e S. Ca e $\mathrm{Mg}$ são elementos contidos de forma abundante nas estruturas lenhosas das espécies florestais. Por isso, ficam imobilizados nas cascas e nos trocos e, consequentemente, apresentam renovação lenta (VITOUSEK, 1982). Por essa razão, Ca foi considerado o segundo elemento com maior concentração e conteúdo na serapilheira (Figura 2 e Tabela 1, respectivamente).

$\mathrm{O}$ conteúdo de $\mathrm{N}$ foi superior nas áreas de indução de regeneração natural e fragmento florestal. Nesses ecossistemas, houve predomínio de Croton matourensis, espécie pioneira que pode ter contribuído para a maior transferência desse elemento para o solo.

$\mathrm{K}$ é um elemento móvel, de liberação rápida da serapilheira para o solo, porém muito susceptível ao processo de lixiviação. Nesse sentido, o menor conteúdo de K em PM em relação a RN e FF está relacionado à morte natural de alguns indivíduos plantados observados durante o experimento. Isso ocasionou exposição do solo e, consequentemente, intensa lixiviação desse nutriente.

Fe e Mn apresentaram altas concentrações na área de regeneração natural em relação ao plantio de mudas arbóreas. Esses resultados podem estar relacionados à possível tolerância e adaptabilidade das espécies vegetais a esses elementos no solo, devolvendo-os para o próprio solo em grande quantidade. Fe e Mn são os micronutrientes mais abundantes na serapilheira, pois, segundo Malavolta et al. (1997), assim como o Ca, estão ligados à estrutura das plantas. Eles variam consideravelmente entre os órgãos vegetais e entre as espécies, assim como durante o período vegetativo.

\section{CONCLUSÕES}

- A indução da regeneração natural foi o método mais eficiente de restauração de áreas degradadas pela mineração de bauxita quanto à deposição de serapilheira, concentração e conteúdo de nutrientes em relação ao plantio de mudas arbóreas nativas.

- A serapilheira e os nutrientes são bons indicadores de monitoramento de restauração de áreas degradadas, demostrando a situação bioquímica dos ecossistemas em desenvolvimento.

\section{AGRADECIMENTOS}

Agradecemos a Mineração Paragominas - MPSA por conceder a área de estudo e todo apoio logístico para execução do trabalho.

\section{REFERENCIAS}

GODINHO, T. O.; CALDEIRA, M. V. W.; ROCHA, J. H. T.; CALIMAN, J. P.; TRAZZI P. A. Quantificação de biomassa e nutrientes da serapilheira acumulada em trecho de Floresta Estacional Semidecidual Submontana, ES. Cerne, Lavras, v. 20, n. 1, p. 11-20, 2014.

GOMES, A. C. S.; LUIZÃO, F. J. Leaf and soil nutrients in a chronosequence of second-growth forest in central Amazonia: Implications for restoration of abandoned lands. Restoration Ecology, Washington, v. 20, n. 3, p. 339-345, 2011.

GUARIGUATA, M. R.; OSTERTAG, R. Neotropical secondary forest succession: changes in structural and functional characteristics. Forest Ecology and Management, Amsterdam, v. 148, p. 185-206, 2001. 
GUIMARÃES, S.; MARTINS, S. V.; NERI, A. V.; GLERIANI, J. M.; SILVA, K. A. Banco de sementes de áreas em restauração florestal em Aimorés, MG. Pesquisa Florestal Brasileira, Colombo, v. 34, n. 80, p. 357 368, 2014.

HYDRO. Hydro Paragominas: Mina de bauxita. 2015. Disponível em: < http://www.hydro.com/pt/A-Hydrono-Brasil/Operacoes-no-Brasil/Hydro-Paragominas-mina/>. Acesso em 31 de julho de 2015.

INMET. Instituto Nacional de Meteorologia. $2^{\circ}$ Distrito de Meteorologia, consulta genérica. Estação automática A202 Paragominas/PA. Serviço Nacional de Informações Hidro-Meteorológicas - SIM. Disponível em: http://www.inmet. gov.br/climatologia. Acesso em 20 out. 2015.

JESUS, E. M.; SANTOS, T. S.; RIBEIRO, G. T.; ORGE, M. D. R.; AMORIM, V. O.; BATISTA, R. C. R. C. Regeneração natural de espécies vegetais em jazidas revegetadas. Floresta e Ambiente, Seropédica, v. 23, n. 2, p. 191-200, 2016.

KAUANO, E. E.; CARDOSO, F. C. G.; TOREZAN, J. M. D.; MARQUES, M. C. M. Micro- and meso-scale factores affect the restoration of Atlantic Forest. Natureza \& Conservação, Rio de Janeiro, v. 11, n.2, p.145151, 2013.

KIM, Y.; KNOX, R. G.; LONGO, M.; MEDVIGY, D.; HUTYRA, L. R.; PYLE, E. H.; WOFSY, S. C.; BRAS, R. L.; MOORCROFT, P. R. Seasonal carbon dynamics and water fluxes in an Amazon rainforest. Global change biology, Urbana-Champaign, v. 18, n. 4, p. 01-11, 2012.

LIMA, T. M.; NEVES, C. A. R. Departamento Nacional de Produção Mineral. Sumário Mineral. Brasília: DNPM, 2014. $152 \mathrm{p}$.

LONDE, V.; SOUSA, H. C.; KOZOVITS, A. R. Litterffal as an indicator of productivity and recovery of ecological functions in a rehabilitated riparian forest at Das Velhas River, southeast Brazil. Tropical Ecology, v. 57, n. 2, p. 355-360, 2016.

MACHADO, D. L.; PEREIRA, M. G.; CORREIA, M. E. F.; DINIZ, A. R.; SANTOS, L. L.; MENEZES, C. E. G. Ciclagem de nutrientes em diferentes estágios sucessionais da mata atlântica na bacia do Rio Paraíba do Sul, RJ. Bioscience jornal, Uberlândia, v. 31, n. 4, p. 1222-1237, 2015.

MACHADO, M. R.; SAMPAIO, P. T. B.; FERRAZ, J.; CAMARA, R.; PEREIRA, M, G.; Nutrient retranslocation in forest species in the Brazilian Amazon. Acta Scientiarum, Maringá, v. 38, n. 1, p. 93-101, 2016.

Malavolta, E.; VITti, G. C.; OliveirA, S. A. Avaliação do Estado Nutricional das Plantas. 2. ed. Piracicaba: Associação Brasileira para Pesquisa de Potassa e do Fosfato, 1997. 319 p.

PAUDEL, E.; DOSSA, G. G. O.; XU, J.; HARRISON, R. D. Litterfall and nutrient return along a disturbance gradient in a tropical montane forest. Forest Ecology and Management, Amsterdam, v. 353, n. 1, p. 97-106, 2015.

QUESADA, C. A.; LLOYD, J.; ANDERSON, L. O.; FYLLAS, N. M.; SCHWARZ, M.; CZIMCZIK, C. I. Soils of Amazonian with particular reference to the RAINFOR sites. Biogeosciences, v. 8, p.1415-1440, 2011.

RODRIGUES, B. D.; MARTINS, S. V.; LEITE HG. Avaliação do potencial da transposição da serapilheira e do banco de sementes do solo para restauração florestal em áreas degradadas. Revista árvore, Viçosa, v. 34, n. 1, p. $65-73,2010$.

RODRIGUES, T. E.; SIlVA, R. C.; SIlVA, J. M. L.; OLIVEIRA JÚNIOR, R. C.; GAMA, J. R. N. F.; VALENTE, M. A. Caracterização e classificação dos solos do município de Paragominas, Estado do Pará. Belém: Embrapa Amazônia Oriental, 2003. 49 p. (Documentos n. 162).

SANCHES, L.; VALENTINI, C. M. A.; PINTO JÚNIOR, O. B.; NOGUEIRA, J. S.; VOURLITIS, G. L.; BIUDES, M. S.; SILVA, C. J.; BAMBI, P.; LOBO, F. A. Seasonal and interannual litter dynamics of a tropical semideciduous forest of the southern Amazon Basin, Brazil. Journal of geophysical research, Washington, v. 113, n. 4, p. 1-9, 2008.

SELLE, G. L. Ciclagem de nutrientes em ecossistemas florestais. Bioscience journal, Uberlândia, v. 23, n. 4, p. 29-39, 2007. 
SOUZA, M. C. R.; PINÃ-RODRIGUES, F. C. M.; CASAGRANDE, J. C.; SILVA, S. F.; SCORIZA, R. N. Funcionalidade ecológica de sistemas agroflorestais biodiversos: uso da serapilheira como indicador da recuperação de áreas de preservação permanente. Floresta, Curitiba, v. 46, n. 1, p. 75-82, 2016.

VILLA, E. B.; PEREIRA, M. G.; ALONSO, J. M.; BEUTLER, S. J.; LELES, P. S. S. Aporte de serapilheira e nutrientes em área de restauração florestal com diferentes espaçamentos de plantio. Floresta e Ambiente, Seropédica, 23, v. 1, p. 90-99, 2016.

VITOUSEK P. Nutrient cycling and nutrient use efficiency. The American Naturalist, Chicago Press, v. 119, n. 4, p. 553-572, 1982.

WANG'ONDU, V. W.; BOSIRE, J. O.; KAIRO, J. G.; KINYAMARIO, J. I.; DAHDOUH-GUEBAS, F.; KOEDAM, N. Litter fall dynamics of restored mangroves (Rhyzophora mucronata Lamk. and Sonneratia alba Sm.) in Kenya. Restoration Ecology, Washington, v. 22, n. 6, p. 824-831, 2014.

ZHANG, H.; YUAN, W.; DONG, W.; LIU, S. Seasonal patterns of litterfall in forest ecosystem worldwide. Ecological Complexity, v. 20, p. 240-247, 2014. 
FLORESTA, Curitiba, PR, v. 48, n. 1, p. 37-48, jan./mar.2018.

Martins.W. B. R. et al. 